\title{
Propofol suppresses gastric cancer tumorigenesis by modulating the circular RNA-PVT1/miR-195-5p/E26 oncogene homolog 1 axis
}

\author{
HONGYANG SUI ${ }^{1}$, CHANGJU ZHU ${ }^{1}$, ZHENZHEN LI $^{2}$ and JIANJUN YANG ${ }^{3}$ \\ Departments of ${ }^{1}$ Emergency, ${ }^{2}$ Medical Biology and ${ }^{3}$ Anesthesiology, \\ The First Affiliated Hospital of Zhengzhou University, Zhengzhou, Henan 450052, P.R. China
}

Received October 28, 2019; Accepted May 22, 2020

DOI: $10.3892 /$ or.2020.7725

\begin{abstract}
Propofol is frequently used for anesthesia in cancer surgery. It has been suggested that treatment with propofol serves a tumor-suppressing role in human gastric cancer (GC). Therefore, the present study aimed to explore the potential mechanism of propofol in GC. In the present study, GC cell lines (HGC-27 and AGS) were treated with various concentrations of propofol, and an MTT assay was performed to detect the cell viability. In addition, flow cytometry and Transwell assays were used to evaluate the apoptosis and invasive ability of GC cells, respectively. Western blotting was performed to detect the protein levels of cyclin-dependent kinase inhibitor P21 (P21), B-cell lymphoma-2 (Bcl-2), matrix metalloproteinase 9 (MMP9) and E26 oncogene homolog 1 (ETS1). Furthermore, reverse transcription-quantitative PCR was used to examine the expression levels of circular RNA-PVT1 (circ-PVT1), micoRNA-195-5p (miR-195-5p) and ETS1 in GC tissues and cells. The target interaction between miR-195-5p and circ-PVT1 or ETS1 was predicted through bioinformatics analysis, and verified by dual-luciferase reporter gene assay. In addition, a mouse xenograft model was established for in vivo experiments. It was identified that propofol inhibited the viability and invasion, but promoted apoptosis of HGC-27 and AGS cells in a dose-dependent manner. Propofol could inhibit Bcl-2 and MMP9 expression, and increase P21 expression in GC cells. The expression levels of circ-PVT1 and ETS1 were increased in GC tissues and cells, and miR-195-5p was decreased. Additionally, the treatment with propofol could lead to decreased circ-PVT1 expression in GC cells. These results could be reversed by ETS1 upregulation or miR-195-5p-knockdown in GC cells. Furthermore, circ-PVT1 could act as a miR-195-5p sponge to modulate ETS1 expression. Additionally, the effects of transfection
\end{abstract}

Correspondence to: Dr Changju Zhu, Department of Emergency, The First Affiliated Hospital of Zhengzhou University, 50 Jianshe East Road, Erqi, Zhengzhou, Henan 450052, P.R. China

E-mail:yxehzv@163.com

Key words: gastric cancer, propofol, circular RNA-PVT1, microRNA-195-5p, E26 oncogene homolog 1 with circ-PVT1 small interfering RNA (si-circ-PVT1) on HGC-27 and AGS cells could be reversed by treatment with miR-195-5p inhibitor. Meanwhile, miR-195-5p inhibitor reversed the si-circ-PVT1-induced low expression of ETS1. Downregulation of ETS1 induced by propofol in HGC-27 and AGS cells could be restored by circ-PVT1 upregulation or miR-195-5p silencing. Circ-PVT1 silencing facilitated the propofol-induced anti-GC effect in vivo. In conclusion, the present study indicated that propofol inhibited the proliferation and invasion, but enhanced the apoptosis of GC cells by regulating the circ-PVT1/miR-195-5p/ETS1 axis.

\section{Introduction}

Propofol is a commonly used anesthetic in the clinic (1). Previous studies have investigated the inhibitory effect of propofol on cancer cells in a variety of tumors, such as breast (2), lung (3) pancreatic (4), ovarian (5), hepatic (6) and gastric cancer (GC) $(7,8)$. In GC, Yang et al (7) reported that propofol suppressed the proliferation of SGC-7901 and MGC-803 cells by promoting inhibitor of growth 3 (7). Peng and Zhang (8) indicated that propofol inhibited proliferation, but induced apoptosis of SGC-7901 cells by regulating matrix metalloproteinase (MMP)2 (8). These previous studies have mostly been limited to the pharmacodynamic effects of propofol on phenotypes, and the underlying mechanisms of the antitumor effects still need to be explored.

Circular RNAs (circRNAs) are a series of endogenous non-coding RNAs with a covalently closed loop structure (9). A previous study confirmed that circRNAs were more stable compared with linear mRNAs due to the lack of a 5'cap and 3'polyadenylate tail (10). There is evidence that circRNAs are specifically expressed in hematological malignancies or certain developmental stages, indicating that circRNAs have considerable regulatory potential by serving as post-transcriptional regulators $(11,12)$. CircRNA-PVT1 (circ-PVT1) has been reported to function as an oncogenic circRNA in tumors, such as oral squamous cell carcinoma, head and neck squamous cell carcinoma, and osteosarcoma (13-15). Circ-PVT1 is highly expressed in GC tissues and cells, and is also associated with poor prognosis; therefore, Chen et al (16) predicted that circ-PVT1 was a novel proliferative factor and prognostic indicator in GC. However, to the best of our knowledge, there are few reports on the mechanism of circ-PVT1 in regulating 
the proliferation, apoptosis and invasion of GC cells (17). In addition, the association between propofol and circ-PVT1 has not been reported.

Accumulating evidence has indicated that microRNAs (miRNAs or miRs) act as regulators by regulating oncogene expression in malignant cancers (18-20). Studies have reported that miR-195-5p is downregulated in GC tissues and cells, and as a tumor suppressor, miR-195-5p can inhibit cell proliferation, invasion and resistance in GC cells (21-23). A previous study revealed that the expression of miR-195-5p in the tissues and serum of patients with GC was associated with tumor diameter, tumor-node-metastasis stage and differentiation (24). In addition, miR-195-5p overexpression can suppress the proliferation and invasion, and promote the apoptosis of GC cells (24). In another study by Wang et al (23), it was identified that the expression level of miR-195-5p was negatively correlated with basic fibroblast growth factor (bFGF) in human GC tissues. In addition, miR-195-5p inhibited the migration and invasion of SNU-1 and KATO-3 cells by downregulating bFGF (23). Nevertheless, the regulatory role of miR-195-5p in GC has not been fully clarified.

E-26 factor has been shown to play an essential role in numerous biological processes, such as proliferation, differentiation, transformation and apoptosis (25-29). E26 oncogene homolog 1 (ETS1) is a gene associated with invasion and metastasis in tumorigenesis via transcriptional factors (30). ETS1 has been shown to mediate MMPs and integrins in numerous types of cancer cells and tissues (31-36). For example, the upregulation of ETS1 can promote invasion and progression of breast cancer by regulating MMP-9 (37).

Considering the aforementioned studies, the present study was designed to improve understanding of the molecular mechanism of propofol in inhibiting GC progression, as well as the associations among circ-PVT1, miR-195-5p and ETS1.

\section{Materials and methods}

Sample collection. The present study received approval from the Ethics Committee of The First Affiliated Hospital of Zhengzhou University (Zhengzhou, China). A total of $30 \mathrm{GC}$ tissue and paired adjacent tissue samples (located $>5 \mathrm{~cm}$ away from the tumor) were collected from patients with GC who underwent total gastrectomy at The First Affiliated Hospital of Zhengzhou University between June 2015 and March 2018. A total of 30 patients were included in the study, including 10 females and 20 males, with an age range of 25-75 years, and the median age at the time of diagnosis was 53 years. The main inclusion criteria were: i) Patients who were diagnosed with GC through pathological examination; ii) patients who were diagnosed and treated for GC for the first time; and iii) patients who were willing to join the study. The exclusion criteria were: i) History of other malignant disease; and ii) recurrent or treated GC. Written informed consent was obtained from each patient before chemotherapy. All samples were collected and stored at $-80^{\circ} \mathrm{C}$.

Cell culture and propofol administration. The human gastric mucosa cell line (GES-1) and GC cell lines (HGC-27, AGS, SNU5 and MKN-45) were obtained from Shanghai Berry Innovation Biotechnology Co., Ltd. (Shanghai, China). All cells were cultured in 90\% Dulbecco's modified Eagle's medium (DMEM; Gibco; Thermo Fisher Scientific, Inc.) with $10 \%$ fetal bovine serum (FBS; Gibco; Thermo Fisher Scientific, Inc.) at $37^{\circ} \mathrm{C}$ with $5 \% \mathrm{CO}_{2}$. The HGC-27 and AGS cells were divided into the following groups: i) Control group, without propofol; ii) $2.5 \mu \mathrm{g} / \mathrm{ml}$ propofol (Sigma-Aldrich; Merck KGaA) group; iii) $5 \mu \mathrm{g} / \mathrm{ml}$ propofol group; and iv) $10 \mu \mathrm{g} / \mathrm{ml}$ propofol group, which were treated for 24,48 and $72 \mathrm{~h}$ at $37^{\circ} \mathrm{C}$. For subsequent experiments, $\mathrm{HGC}-27$ and AGS cells were exposed to $5 \mu \mathrm{g} / \mathrm{ml}$ propofol for $48 \mathrm{~h}$ at $37^{\circ} \mathrm{C}$.

MTT assay. The viability of GC cells was evaluated by MTT assay. Briefly, HGC-27 and AGS cells $\left(3 \times 10^{3} /\right.$ well) with or without transfection were seeded into 96-well plates (Corning, Inc.) and maintained in an incubator with $5 \% \mathrm{CO}_{2}$ at $37^{\circ} \mathrm{C}$ for 24 h. Then, MTT (20 $\mu$; Sigma-Aldrich; Merck KGaA) was added to each well for $4 \mathrm{~h}$. Subsequently, the supernatant of each well was discarded and DMSO (150 $\mu \mathrm{l}$; Sigma-Aldrich; Merck KGaA) was added to dissolve the formazan crystals. Finally, a microplate absorbance reader (Thermo Fisher Scientific, Inc.) was used to measure the optical density value at $490 \mathrm{~nm}$.

Flow cytometry assay. Annexin V-FITC/PI Apoptosis Detection kit (Vazyme Biotech Co., Ltd.) was used for the flow cytometry assay. In brief, cells (1x10 $/$ well) were seeded into 6-well plates, treated with $0.25 \%$ trypsin and washed with pre-cooled PBS. Subsequently, $2 \times 10^{5}$ cells were resuspended in $100 \mu \mathrm{l} 1 \mathrm{X}$ binding buffer and then incubated with $5 \mu \mathrm{l}$ Annexin V-FITC and PI staining solution for $10 \mathrm{~min}$ at room temperature without light. Finally, the apoptotic cells were assessed using a flow cytometer (BD Biosciences).

Transwell assay. The rate of cell invasion was investigated using a Transwell chamber (Corning Inc.) coated with Matrigel at $4^{\circ} \mathrm{C}$ overnight. The lower chamber was filled with DMEM with $10 \%$ FBS, while the transfected HGC-27 and AGS cells $\left(1 \times 10^{5} /\right.$ well) were added to the upper chamber with $100 \mu \mathrm{l}$ serum-free medium. After incubation for $24 \mathrm{~h}$ at $37^{\circ} \mathrm{C}$, paraformaldehyde (Sigma-Aldrich; Merck KGaA) was used to attach cells located on lower surface of the upper chamber for $20 \mathrm{~min}$ at the room temperature. Cells were analyzed under a fluorescence microscope (magnification, x100) after staining with crystal violet for $30 \mathrm{~min}$ at the room temperature.

Western blotting. Western blotting was performed as described previously (38). Briefly, protein samples were extracted with RIPA lysis buffer (Thermo Fisher Scientific, Inc.) and then quantified by BCA Protein assay kit (Thermo Fisher Scientific, Inc.). Subsequently, each protein sample (50 $\mu \mathrm{g} / \mathrm{lane})$ was separated by $10 \%$ SDS-PAGE and then transferred onto polyvinylidene fluoride membranes (EMD Millipore). Then, the membranes were incubated with primary antibodies overnight at $4^{\circ} \mathrm{C}$ after blocking with $5 \%$ non-fat milk for $1 \mathrm{~h}$ at room temperature. The next day, the corresponding secondary antibody was used for $1 \mathrm{~h}$ at room temperature, and the signals were visualized using an enhanced chemiluminescence kit (EMD Millipore). Primary antibodies against the following were used: Cyclin-dependent kinase inhibitor P21 (P21; catalog no. ab109520; 1:1,000; Abcam), B-cell lymphoma-2 
(Bcl-2; catalog no. ab32124; 1:1,000; Abcam), MMP9 (catalog no. ab76003; 1:2,500; Abcam), ETS1 (catalog no. ab26096; 1:2,500; Abcam) and GAPDH (catalog no. ab9485; 1:2,500; Abcam). In addition, the horseradish peroxidase-conjugated goat anti-rabbit secondary antibody (catalog no. ab205718; 1:5,000; Abcam) was detected with the enhanced chemiluminescence kit and analyzed with Quantity One v4.6.2 software (Bio-Rad Laboratories, Inc.).

Reverse transcription-quantitative PCR (RT-qPCR). Total RNA from HGC-27 and AGS cells was extracted using TRIzol reagent (Thermo Fisher Scientific, Inc.). Primer-Script RT-PCR kit (Takara Bio, Inc.) or miRNA Reverse Transcription kit (GeneCopoeia, Inc.) was used, according to the manufacturers' protocols, to synthesize the first-strand complementary DNA of miR-195-5p and circ-PVT1 or ETS1, respectively. The levels of miR-195-5p and circ-PVT1 or ETS1 were assessed via SYBR Premix Dimer Eraser kit (Takara Bio, Inc.). The thermocycling conditions were as follows: Denaturation at $95^{\circ} \mathrm{C}$ for $10 \mathrm{~min}$, followed by 40 cycles of denaturation at $95^{\circ} \mathrm{C}$ for $30 \mathrm{sec}$, annealing at $60^{\circ} \mathrm{C}$ for $30 \mathrm{sec}$ and extension at $72^{\circ} \mathrm{C}$ for $1 \mathrm{~min}$. The primer sequences used were as follows: circ-PVT1 forward, 5'-GGTTCCACCAGCGTTATTC-3' and reverse, 5'-CAACTTCCTTTGGGTCTCC-3'; miR-195-5p forward, 5'-CGTAGCAGCACAGAAAT-3' and reverse, 5'-GTGCAG GGTCCGAGGT-3'; ETS1 forward, 5'-AGCCGACTCTCA CCATCATC-3' and reverse, 5'-CAAGGCTTGGGACATCAT TT-3'; U6 forward, 5'-GCTTCGGCAGCACATATACTAAA AT-3' and reverse, 5'-CGCTTCACGAATTTGCGTGTCAT-3'; and GAPDH forward, 5'-CAATGACCCCTTCATTGACC-3' and reverse, 5'-TGGAAGATGGTGATGGGATT-3'. The levels of miR-195-5p, circ-PVT1 and ETS1 were calculated by the $2^{-\Delta \Delta \mathrm{Cq}}$ method (39), and GAPDH or U6 were used as an internal control for circ-PVT1 and miR-195-5p or ETS1, respectively.

Cell transfection. For circRNA downregulation, small interfering RNA against circ-PVT1 (si-circ-PVT1; 3'-CUGUCA GCUGCAUGGAGCUUCGU-5') and its negative control (si-NC; 3'-AAUUCUCCGAACGUGUCACGU-5') were constructed by Shanghai Genepharma Co., Ltd.. For circ-PVT1 upregulation, circ-PVT1 sequences were amplified by PCR and inserted into a pcDNA vector (Invitrogen; Thermo Fisher Scientific, Inc.) to generate fusion plasmids, and a pcDNA empty vector was used as the control. For miR-195-5p enrichment or inhibition, miR-195-5p mimic (5'-UAGCAGCACAGA AAUAUUGGC-3'), miR-195-5p inhibitor (anti-miR-195-5p: 5'-GCCAAUAUUUCUGUGCUGCUA-3') and the negative controls (miR-NC, 5'-UCACAACCUCCUAGAAAGAGU AGA-3'; and anti-miR-NC, 5'-CAGUACUUUUGUGUAGUA CAA-3') were purchased from Guangzhou RiboBio Co., Ltd.. All plasmids $(2 \mu \mathrm{g})$ and oligonucleotides (40 $\mathrm{nM})$ were transfected into HGC-27 and AGS cells using Lipofectamine 3000 (Invitrogen; Thermo Fisher Scientific, Inc.). At $48 \mathrm{~h}$ post-transfection, HGC-27 and AGS cells were collected and used for further analyses.

Bioinformatics analysis and dual-luciferase reporter assay. StarBase v2.0 online software (http://starbase.sysu.edu.cn/starbase2/) was used to predict the potential binding relationship between miR-195-5p and circ-PVT1 or ETS1. The associations between miR-195-5p and circ-PVT1 or ETS1 were verified by dual-luciferase reporter assay. In brief, the sequences of circ-PVT1 wild type (WT) containing the binding sites with miR-195-5p and corresponding circ-PVT1 mutant (MUT) sequences were amplified and cloned into the pRL-CMV vector (Promega Corporation), and termed WT-circ-PVT1 and MUT-circ-PVT1, respectively. Subsequently, WT-circ-PVT1 and MUT-circ-PVT1 were introduced into HGC-27 and AGS cells together with miR-195-5p or miR-NC using Lipofectamine 3000 (Invitrogen; Thermo Fisher Scientific, Inc.). Following $48 \mathrm{~h}$, the luciferase activity was detected using a Dual-Luciferase Reporter assay kit (Promega Corporation). Similarly, ETS1-3'UTR-WT containing the binding site for miR-195-5p and ETS1-3'UTR-MUT were also constructed and used for luciferase activity analysis, according to the previous method. Renilla luciferase activities were used as the internal control for the normalization of firefly luciferase activity.

Xenograft tumor model. Firstly, a sh-circ-PVT1 lentivirus plasmid was generated by transfecting a shRNA sequence of circ-PVT1 (5'-GAATGCCTCATGGATTCTTAC-3') into a pLKO.1 lentivirus vector (Addgene, Inc.). A pLKO.1 empty vector was used as a negative control (sh-control). Subsequently, sh-circ-PVT1 or sh-control vector (40 nM) was transfected into AGS cells along with the lentivirus packaging plasmids (psPAX2 and pMD2 G; Addgene, Inc.) using Lipofectamine 3000 (Invitrogen; Thermo Fisher Scientific, Inc.), followed by incubation for $72 \mathrm{~h}$. The transfection efficiency of sh-circ-PVT1 was determined by RT-qPCR.

A total of $32 \mathrm{BALB} / \mathrm{c}$ male nude mice (age, 5 weeks, weight 16-22 g) were purchased from Charles River Laboratories Inc., followed by maintenance under specific pathogen-free conditions with a 12-h light/dark cycle, and a constant and suitable temperature $\left(25^{\circ} \mathrm{C}\right)$ and humidity $(60 \%)$, with easy access to food and water. The mice were randomly divided into the following four groups ( $n=8$ per group); i) Sh-control + PBS group; ii) sh-control + propofol group; iii) sh-circ-PVT1 + PBS group; and iv) sh-circ-PVT1 + propofol group. AGS cells $\left(2 \times 10^{6}\right)$ were stably transfected with $40 \mathrm{nM}$ sh-circ-PVT1 (forward, 5'-GATCCGAATGCCTCATGGATTCTTACCTC GAGCATTCTTAGGTACTCCGTAAGTTTTTG-3' and reverse, 5'-AATTCAAAAAGAATGCCTCATGGATTCTTA CCTCGAGCATTCTTAGGTACTCCGTAAGG-3') using Lipofectamine 3000 (Invitrogen; Thermo Fisher Scientific, Inc.), and were injected subcutaneously into the left hind back of nude mice. The control groups received an equal amount of sh-control-transfected cells. After 7 days, $30 \mathrm{mg} / \mathrm{kg}$ propofol or PBS was intraperitoneally injected every 3 days. The tumor volume was measured using a caliper every 4 days. At day 27 post-inoculation, the mice were sacrificed by cervical dislocation following deep anesthesia with $2 \%$ isoflurane (Baxter Healthcare), and tumor tissues were collected to measure the tumor weight and for RT-qPCR. The experiments were approved by the Experimental Animal Ethics Committee of The First Affiliated Hospital of Zhengzhou University (Zhengzhou, China).

Statistical analysis. Data were collected from at least three independent experiments and analyzed by GraphPad Prism 7.0 (GraphPad Software Inc.). Data are presented as the 


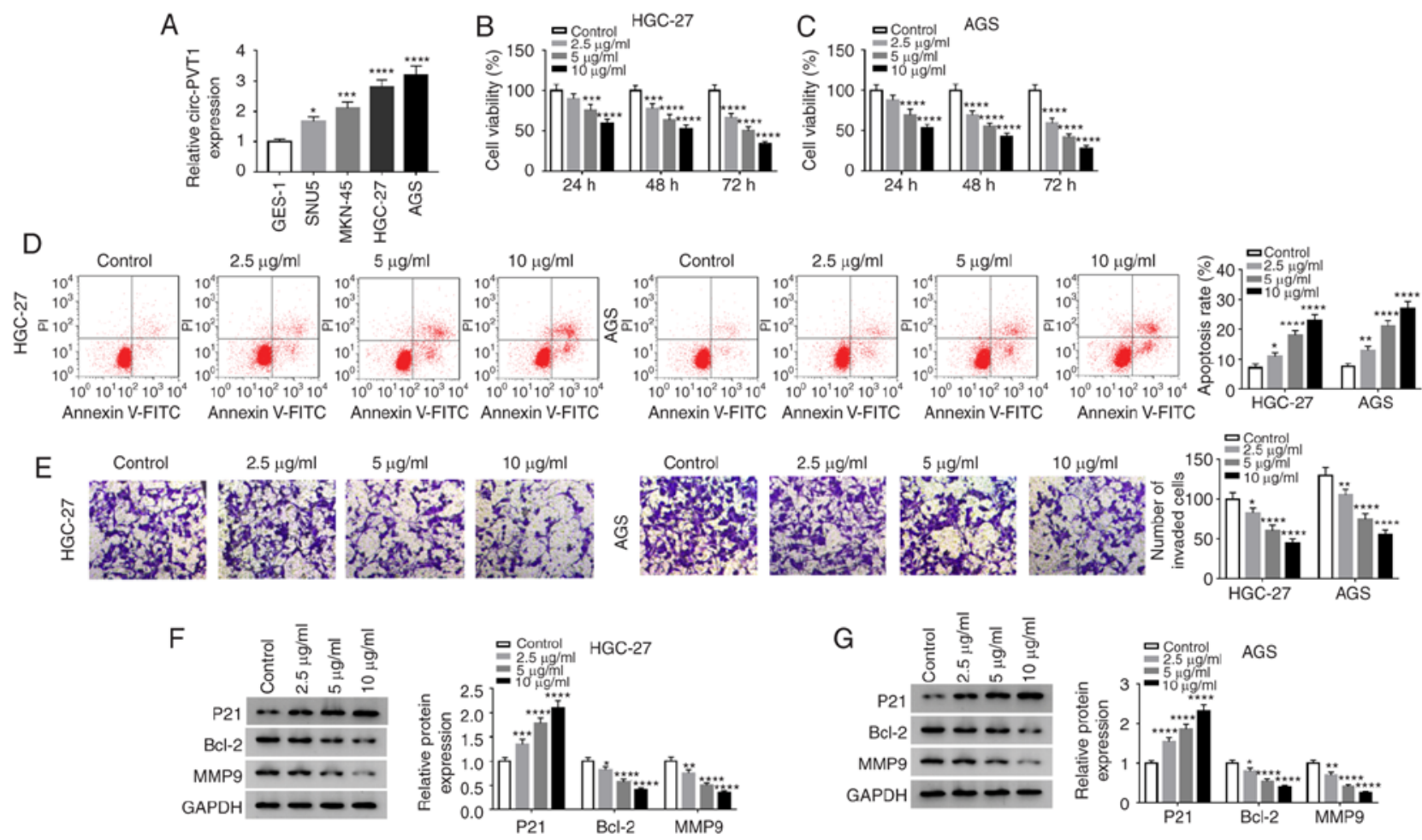

Figure 1. Propofol suppresses the viability and invasion, but induces apoptosis of GC cells. (A) Circ-PVT1 level was detected in a human gastric mucosa cell line (GES-1) and GC cell lines (SNU5, MKN-45, HGC-27 and AGS) by reverse transcription-quantitative PCR. MTT assay was performed to assess the cell viability of (B) HGC-27 and (C) AGS cells at 24, 48 and $72 \mathrm{~h}$ after treatment with $0,2.5,5 \mathrm{or} 10 \mu \mathrm{g} / \mathrm{ml}$; propofol (D) Flow cytometry was performed to measure the rate of apoptosis when cells were treated with propofol. (E) Transwell assay was performed to evaluate the invasion of HGC-27 and AGS cells following treatment with propofol (Magnification, x100). Western blotting was used to detect the expression of P21, Bcl-2 and MMP9 in (F) HGC-27 and (G) AGS cells. ${ }^{*} \mathrm{P}<0.05,{ }^{* *} \mathrm{P}<0.01,{ }^{* * *} \mathrm{P}<0.001,{ }^{* * * * *} \mathrm{P}<0.0001$ vs. control. Circ-PVT1, circular RNA-PVT1; GC, gastric cancer; p21, cyclin-dependent kinase inhibitor $\mathrm{p} 21 ;$ Bcl-2, B-cell lymphoma-2; MMP9, matrix metalloproteinase 9.

mean \pm standard deviation. Pearson's correlation analysis was used to analyze the correlation between expression levels. The analyses between two groups were performed using Student's t-test. One-way analysis of variance followed by Tukey's post-hoc test was used to compare the differences among three or more groups. $\mathrm{P}<0.05$ was considered to indicate a statistically significant difference.

\section{Results}

Propofol suppresses cell viability and invasion, but induces apoptosis of GC cells. The present study detected the expression level of circ-PVT1 in the human gastric mucosa cell line GES-1 and the GC cell lines SNU5, MKN-45, HGC-27 and AGS. As presented in Fig. 1A, circ-PVT1 level was significantly higher in GC cell lines (HGC-27, AGS, SNU5 and MKN-45) compared with GES-1 cells, especially for HGC-27 and AGS cells. Therefore, HGC-27 and AGS cells were selected for further experiments. To validate the function of propofol on the progression of GC cells, an MTT assay was employed to detect the viability of HGC-27 and AGS cells treated with various concentrations of propofol for different durations. Compared with the control group, the viability of HGC-27 and AGS cells treated with propofol significantly decreased in a concentration-dependent manner (Fig. 1B and C). To analyze the rate of cell apoptosis, flow cytometry was performed with cells administrated propofol and the results demonstrated that propofol significantly promoted apoptosis in a dose-dependent manner (Fig. 1D). A Transwell assay was performed to assess the invasive abilities of HGC-27 or AGS cells treated with various concentrations of propofol for $48 \mathrm{~h}$. It was identified that treatment with significantly reduced cell invasion in a concentration-dependent manner compared with the control group (Fig. 1E). Subsequently, western blotting was performed to analyze the protein expression of P21, Bcl-2 and MMP9, which revealed that Bcl-2 and MMP9 expression levels were significantly decreased following treatment with propofol in a dose-dependent manner, whereas the level of P21 was significantly increased as the dose of propofol increased (Fig. 1F and G).

Circ-PVT1 is upregulated in GC tissues and cells, and circ-PVT1 reverses the propofol-mediated effects on $G C$ cells. Next, the potential role of circ-PVT1 in GC was investigated, and RT-qPCR revealed that circ-PVT1 expression was significantly higher in GC tissues (Fig. 2A) and cells (Fig. 2B) compared with the normal controls. In addition, propofol significantly reduced the expression level of circ-PVT1 in a dose-dependent manner in GC cells (Fig. 2C). To further confirm the association between circ-PVT1 and propofol, rescue assays with HGC-27 and AGS cells were performed. As presented in Fig. 2D, circ-PVT1 overexpression reversed the propofol-induced repression of circ-PVT1 in HGC-27 and AGS cells. Circ-PVT1 overexpression also significantly reversed the suppressive effect of propofol on the proliferation (Fig. 2E and F) and invasion (Fig. 2H) of HGC-27 and AGS cells. In addition, circ-PVT1 overexpression significantly 

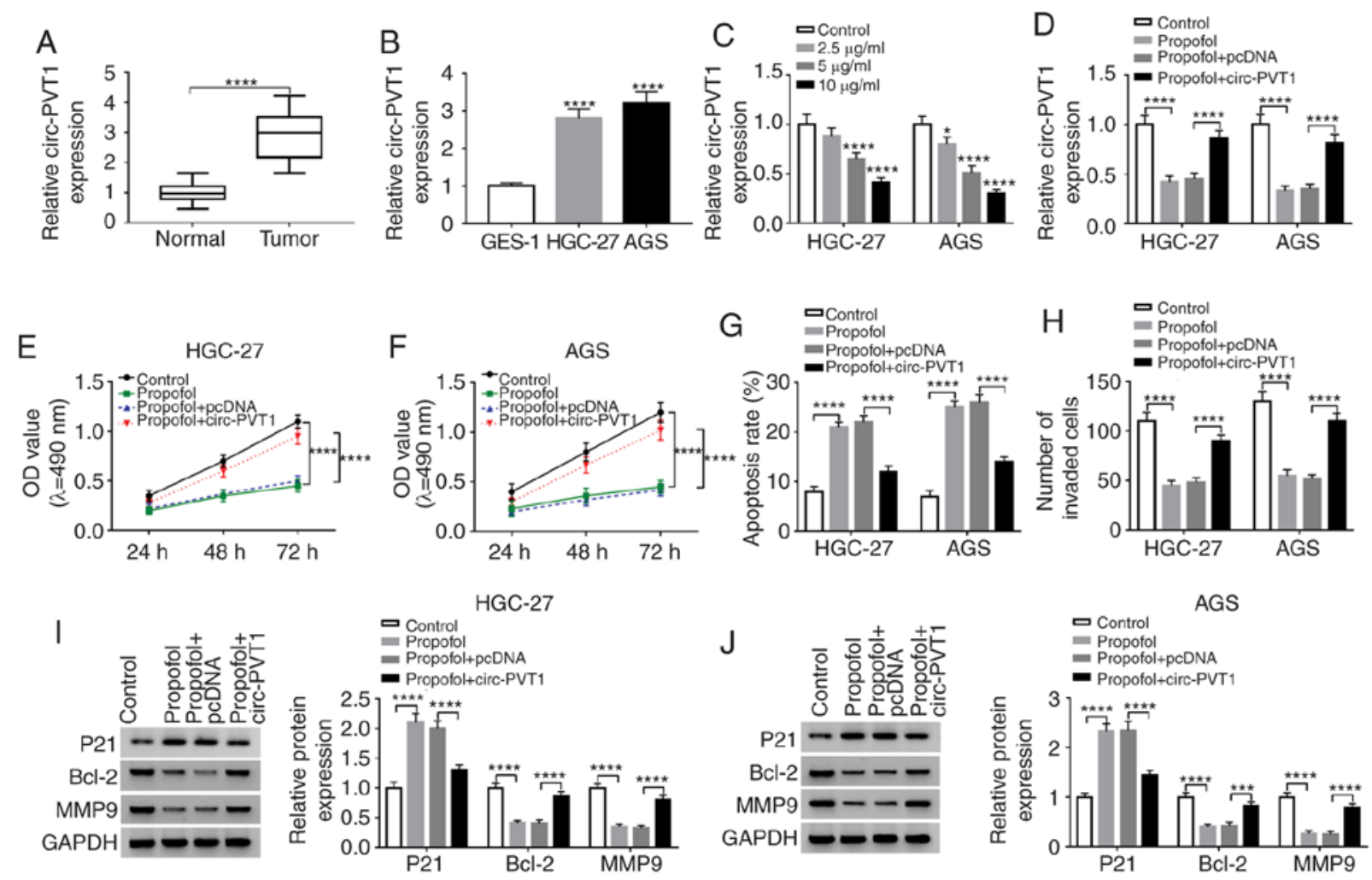

Figure 2. Circ-PVT1 is upregulated in GC tissues and cells, and circ-PVT1 restores the propofol-mediated effects on GC cells. Circ-PVT1 is expressed at a higher level in GC (A) tissues or (B) cells (HGC-27 and AGS) compared with the corresponding controls. (C) Circ-PVT1 level was decreased in a concentration-dependent manner when cells were treated with propofol. (D) Rescued circ-PVT1 expression in HGC-27 and AGS cells following propofol treatment was demonstrated by reverse transcription-quantitative PCR. Rescued cell viability of (E) HGC-27 and (F) AGS cells was investigated by MTT assay. (G) The effect of circ-PVT1 on the apoptosis of HGC-27 and AGS cells was confirmed by flow cytometry assay. (H) The invasion of cells was rescued by circ-PVT1 overexpression as determined by Transwell assay. Western blotting revealed that the expression levels of P21, Bcl-2and MMP9 were restored by circ-PVT1 overexpression in (I) HGC-27 and (J) AGS cells treated with propofol. ${ }^{*} \mathrm{P}<0.05,{ }^{* * * *} \mathrm{P}<0.001,{ }^{* * * * *} \mathrm{P}<0.0001$ vs. control. Circ-PVT1, circular RNA-PVT1; GC, gastric cancer; P21, cyclin-dependent kinase inhibitor P21; Bcl-2, B-cell lymphoma-2; MMP9, matrix metalloproteinase 9; OD, optical density.

reversed the increased apoptotic rate induced by propofol in GC cells (Fig. 2G). Additionally, the aberrant expression levels of P21, Bcl-2 and MMP9 were significantly reversed by circ-PVT1 overexpression in HGC-27 and AGS cells (Fig. 2I and J).

Circ-PVT1 directly binds to miR-195-5p. In order to identify a specific miRNA that is regulated by circ-PVT1, bioinformatics analysis and a dual-luciferase reporter assay were performed. First, the results of bioinformatics analysis with starBase v2.0 suggested that miR-195-5p, which contains the putative binding sites, was a target of circ-PVT1 (Fig. 3A). To further demonstrate that miR-195-5p is a target of circ-PVT1, the dual-luciferase reporter assay was applied. The fragment of circ-PVT1 containing miR-195-5p binding sites (WT-circ-PVT1) or mutant fragment (MUT-circ-PVT1) was inserted into plasmids. Then, cells were co-transfected with plasmid and miR-195-5p or miR-NC. As presented in Fig. 3B and C, the luciferase activity in WT-circ-PVT1-transfected cells was significantly decreased by miR-195-5p. However, there was no change in cells transfected with MUT-circ-PVT1. Subsequently, the expression level of miR-195-5p was measured in tissues and cells, which revealed that miR-195-5p was significantly lower in GC tissues or cells compared with normal controls (Fig. 3D and E). In addition, a significant negative correla- tion was identified between miR-195-5p and circ-PVT1 in $30 \mathrm{GC}$ tumor tissues (Fig. 3F). Subsequently, the level of circ-PVT1 was decreased or increased in HGC-27 and AGS cells by transfection with si-circ-PVT1 or circ-PVT1 vector for loss- and gain-of function, respectively (Fig. 3G). Following transfection, the level of miR-195-5p in GC cells transfected with si-circ-PVT1 or circ-PVT1 vector was assessed, and it was identified that the level of miR-195-5p was significantly increased in circ-PVT1-knockdown HGC-27 and AGS cells, and significantly decreased in circ-PVT1-upregulated HGC-27 and AGS cells (Fig. 3H). In the rescue assays, knockdown of miR-195-5p significantly inhibited the miR-195-5p expression (Fig. 3I), and significantly increased the proliferation (Fig. 3J and K) and invasion (Fig. 3M) of si-circ-PVT1-transfected HGC-27 and AGS cells. Furthermore, miR-195-5p-knockdown significantly reversed the increase in apoptosis rate induced by si-circ-PVT1 transfection (Fig. 3L). Additionally, decreased expression of Bcl-2 and MMP9, and increased expression of P21 induced by circ-PVT1-knockdown could be eliminated by miR-195-5p-knockdown (Fig. 3N and O).

miR-195-5p inhibitor reverses propofol-mediated effects on the proliferation, apoptosis and invasion of GC cells. To investigate the association between miR-195-5p and propofol, RT-qPCR assays demonstrated that miR-195-5p expression was significantly 
A

WT-circ-PVT1 5' UGCCGUGUUUAU--UGCUGCUG 3' miR-195-5p 3' CGGUUAUAAAGACACGACGAU 5'

MUT-circ-PVT1 5' UGGCGAGAAAAA-ACGACGAG $3^{\prime}$
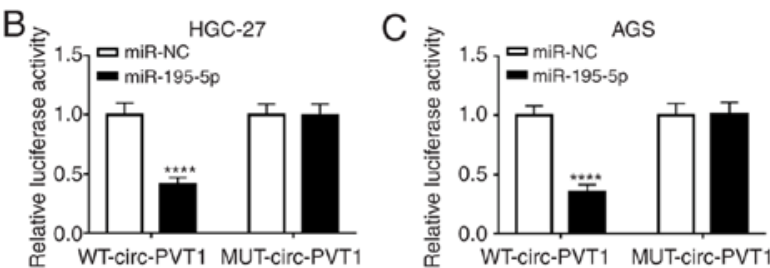

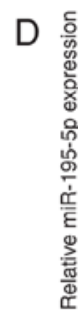

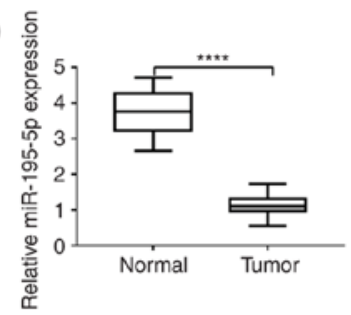

E
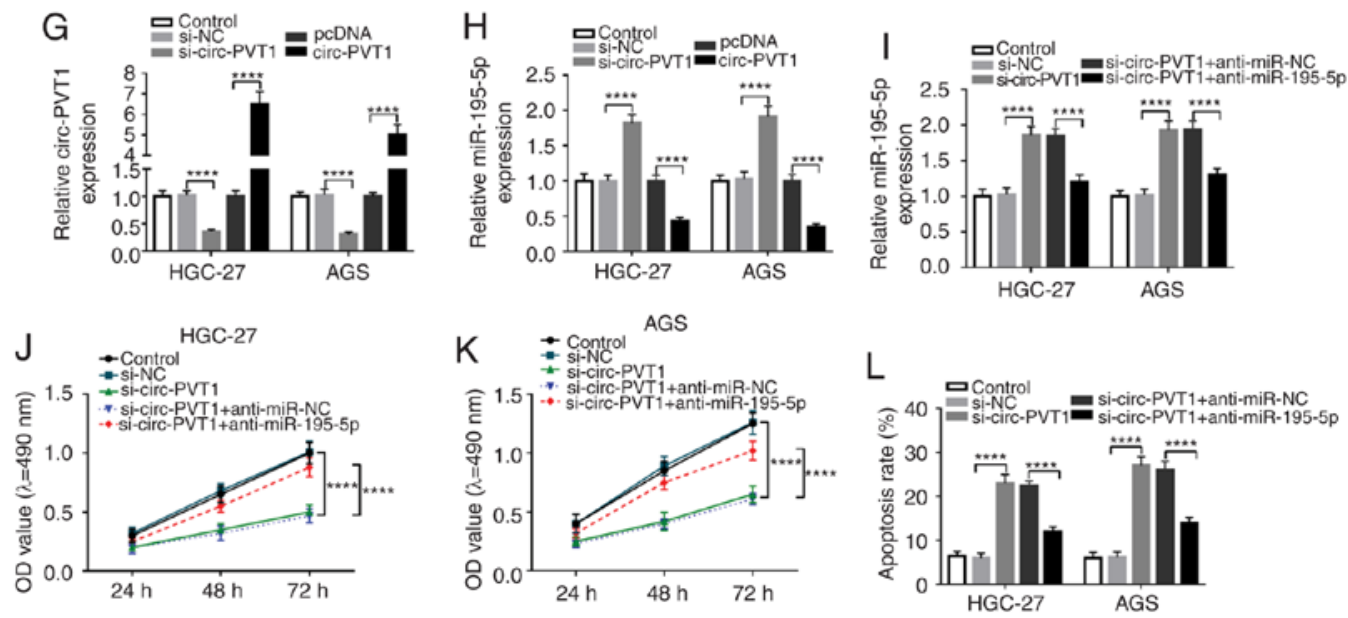
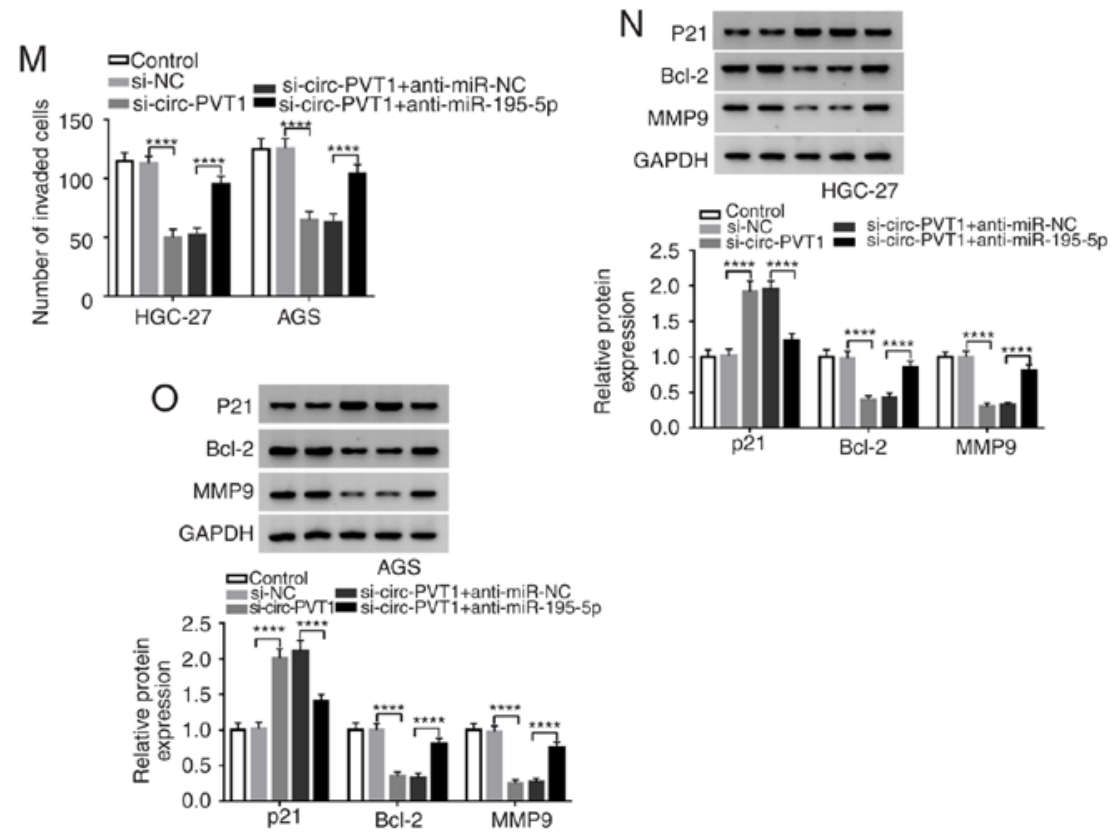

Figure 3. miR-195-5p is a target of circ-PVT1. (A) The putative binding sites between miR-195-5p and circ-PVT1 were predicted by starBase v2.0. The predicted sites were confirmed by dual-luciferase reporter assay with (B) HGC-27 and (C) AGS cells. Expression of miR-195-5p in GC (D) tissues and (E) cells. (F) The correlation between circ-PVT1 and miR-195-5p expression in GC tissues was analyzed by Pearson's correlation analysis. (G) The expression of circ-PVT1 in HGC-27 and AGS cells was detected by RT-qPCR following circ-PVT1-knockdown or overexpression. (H) The expression of miR-195-5p in HGC-27 and AGS cells was detected by RT-qPCR following circ-PVT1-knockdown or overexpression. Anti-miR-195-5p was used for the rescue experiments of si-circ-PVT1. (I) Recovered expression of miR-195-5p was observed following anti-miR-195-5p transfection. The effect of si-circ-PVT1 on viability was reversed by transfection of (J) HGC-27 and (K) AGS cells with anti-miR-195-5p. (L and M) The effect of anti-miR-195-5p on apoptosis was detected by flow cytometry assay. ( $\mathrm{N}$ and $\mathrm{O}$ ) The si-circ-PVT1-induced effects on the expression levels of P21, Bcl-2 and MMP9 were reversed by anti-miR-195-5p, as revealed by western blotting. ${ }^{* * * * *} \mathrm{P}<0.0001$ vs. control. miR-195-5p, microRNA-195-5p; Circ-PVT1, circular RNA-PVT1; GC, gastric cancer; P21, cyclin-dependent kinase inhibitor P21; Bcl-2, B-cell lymphoma-2; MMP9, matrix metalloproteinase 9; OD, optical density; RT-qPCR, reverse transcription-quantitative PCR; anti-miR-195-5p, miR-195-5p inhibitor; WT, wild type; MUT, mutant; NC, negative control. 
A

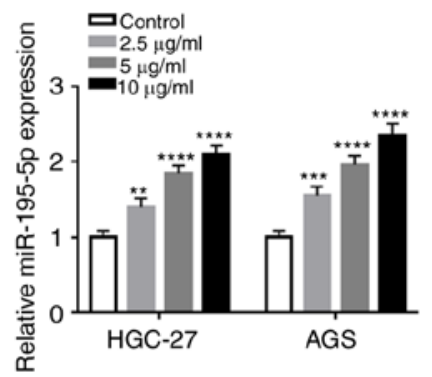

D

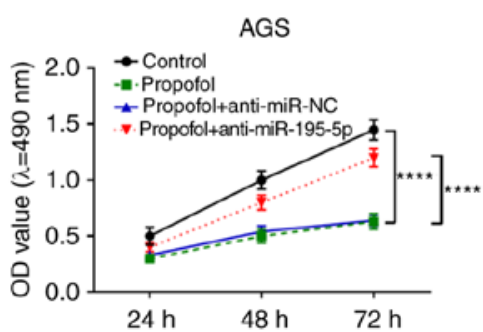

B.

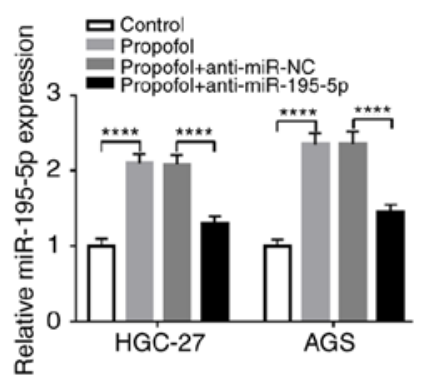

$\mathrm{E}$

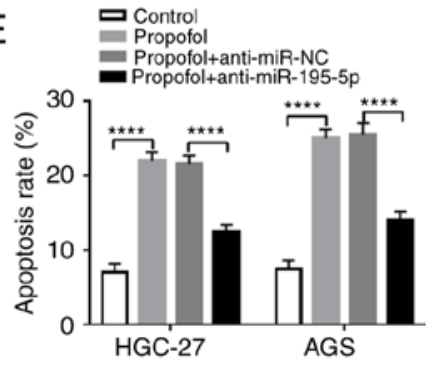

C
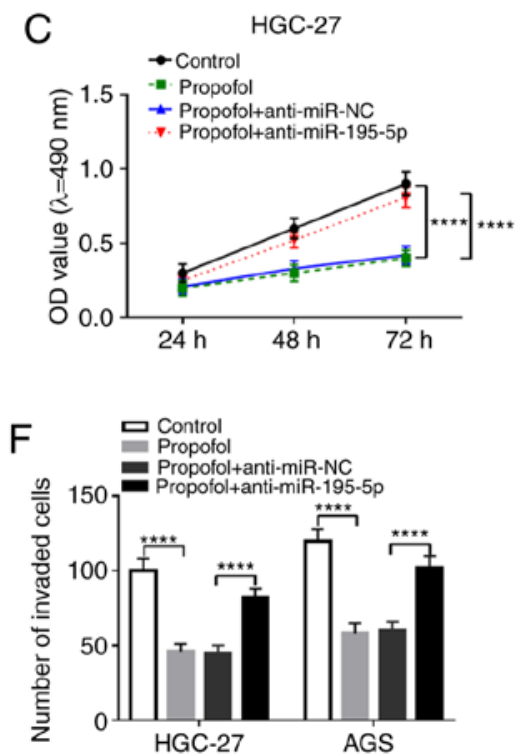
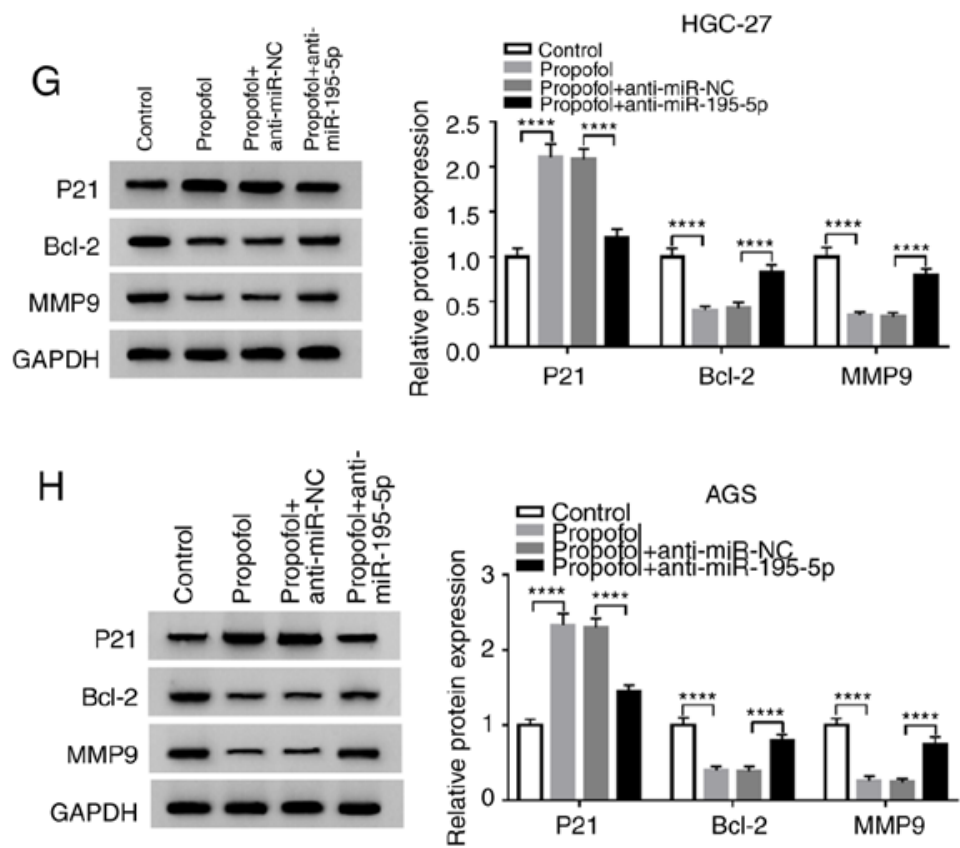

Figure 4. miR-195-5p inhibitor reverses the propofol-mediated effects on the proliferation, apoptosis and invasion of gastric cancer cells. (A) RT-qPCR was used to detect the miR-195-5p expression in cells treated with increasing concentrations of propofol. (B) RT-qPCR was performed to detect the effects of anti-miR-195-5p on the propofol-induced expression of miR-195-5p in HGC-27 and AGS cells. Anti-miR-195-5p revered the propofol-induced effects on the viability of (C) HGC-27 and (D) AGS cells, and the (E) apoptosis and (F) invasion, which was measured by MTT, flow cytometry and Transwell assays, respectively. Western blot assay was performed to assess the effects of anti-miR-195-5p on the propofol-induced changes in the protein expression of P21, Bcl-2 and MMP9 in (G) HGC-27 and (H) AGS cells. ${ }^{* *} \mathrm{P}<0.05,{ }^{* * * *} \mathrm{P}<0.001,{ }^{* * * * *} \mathrm{P}<0.0001$ vs. control. Circ-PVT1, circular RNA-PVT1; P21, cyclin-dependent kinase inhibitor P21; Bcl-2, B-cell lymphoma-2; MMP9, matrix metalloproteinase 9; OD, optical density; miR-195-5p, microRNA-195-5p; RT-qPCR, reverse transcription-quantitative PCR; anti-miR-195-5p, miR-195-5p inhibitor; NC, negative control.

increased as the concentration of propofol increased (Fig. 4A), and that miR-195-5p inhibitor could significantly reverse the increased expression of miR-195-5p induced by propofol in GC cells (Fig. 4B). This indicated that the tumor-suppressive effect of propofol occurred in a miR-195-5p-dependent manner. Subsequently, MTT, flow cytometry and Transwell assays revealed that miR-195-5p inhibitor reversed the effects of propofol on the proliferation (Fig. 4C and D), invasion (Fig. 4F) and apoptosis (Fig. 4E) of HGC-27 and AGS cells. Similarly, the decrease of Bcl-2 and MMP9, and increase of P21 induced by propofol could be significantly reversed by miR-195-5p silencing (Fig. 4G and H).
ETS1 is a direct target of miR-195-5p. StarBase v2.0 was used to identify the potential targets of miR-195-5p on the 3'-UTR of ETS1 mRNA (Fig. 5A). Subsequently, a dual-luciferase activity assay was performed to further confirm the association between miR-195-5p and ETS1. The results demonstrated that the luciferase activity of ETS1-3'UTR-WT was inhibited by miR-195-5p, as no distinct change of ETS1-3'UTR-MUT luciferase activity was detected (Fig. 5B and C). In addition, ETS1 expression was significantly higher in GC tissues and cells compared with normal controls (Fig. 5D-G). Notably, the relative ETS1 expression was significantly negatively correlated with that of 
A

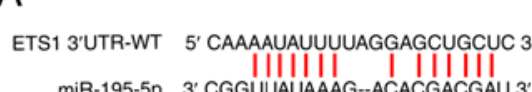

ETS1 3'UTR-MUT 5' CAAUUAUAAAUAGCACGACGAC 3'
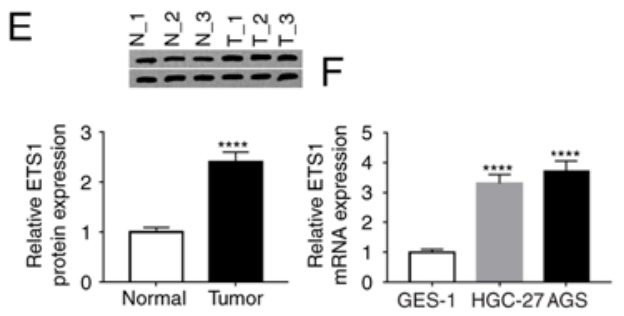

$\mathrm{B}$

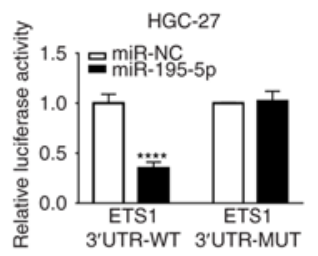

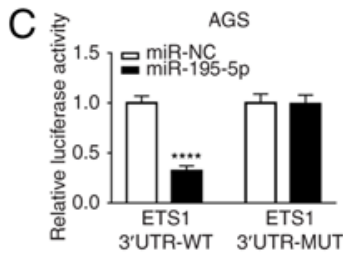
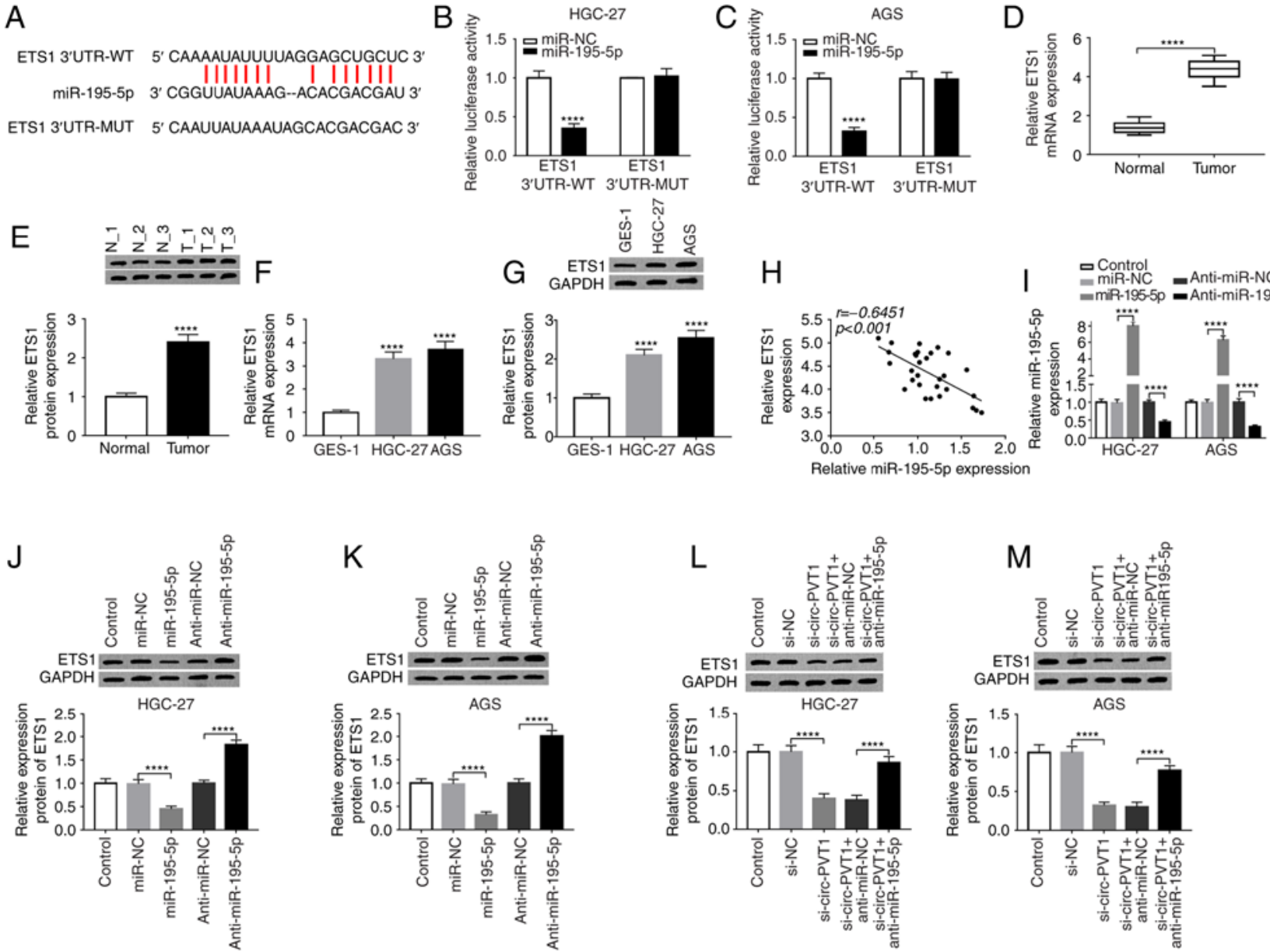

Figure 5. ETS1 is a direct target of miR-195-5p. (A) The putative binding sites between miR-195-5p and circ-PVT1 were predicted by starBase v2.0. The predicted sites were confirmed by dual-luciferase reporter assay with (B) HGC-27 and (C) AGS cells. ETS1 (D) mRNA and (E) protein expression was assessed in gastric cancer tissues by RT-qPCR and western blotting, respectively. ETS1 (F) mRNA and (G) protein expression was assessed in HGC-27 and AGS cells by RT-qPCR and western blotting, respectively. (H) Pearson's correlation analysis was applied to evaluate the correlation between ETS1 and miR-195-5p expression in GC tissues. (I) Expression of miR-195-5p in cells was detected by RT-qPCR following miR-195-5p overexpression or knockdown. The expression of ETS1 in (J) HGC-27 and (K) AGS cells was detected by western blotting after miR-195-5p overexpression or knockdown. The reversed expression of ETS1 was detected by western blotting after (L) HGC-27 and (M) AGS cells were transfected with anti-miR-195-5p. ${ }^{* * * *} \mathrm{P}<0.0001$ vs. control. Circ-PVT1, circular RNA-PVT1; ETS1, E26 oncogene homolog; miR-195-5p, microRNA-195-5p; RT-qPCR, reverse transcription-quantitative PCR; anti-miR-195-5p, miR-195-5p inhibitor; NC, negative control; si, small interfering RNA.

A
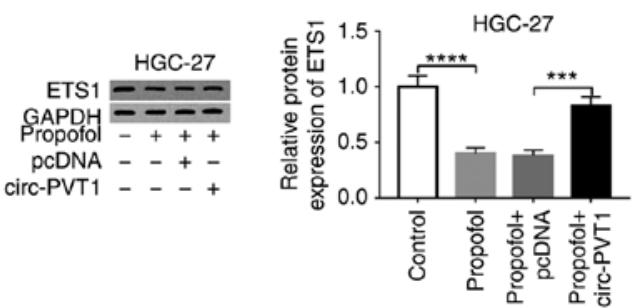

C

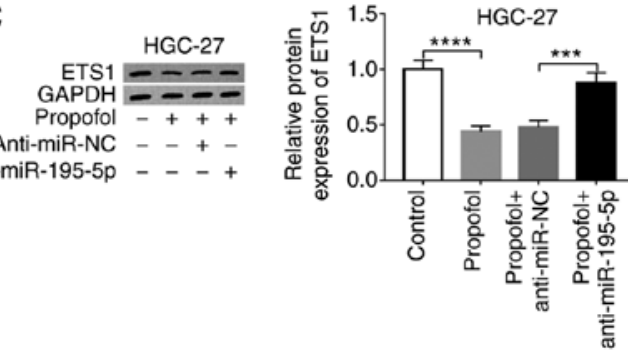

B
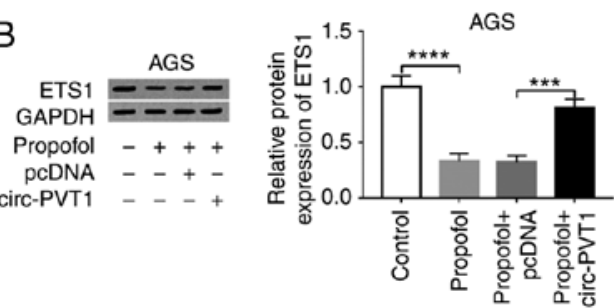

D

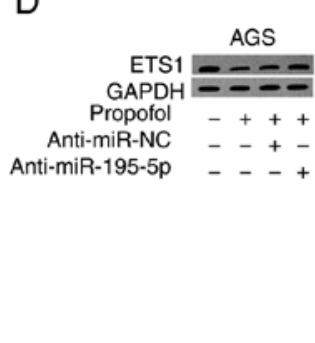

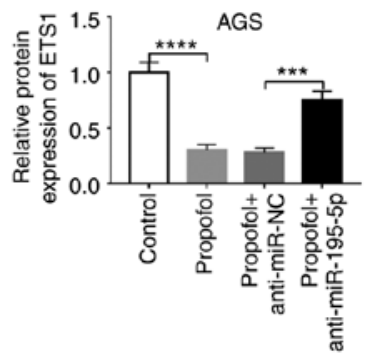

Figure 6. Circ-PVT1 overexpression reversed the propofol-induced effects on ETS1 in GC cells. Circ-PVT1 expression reversed the propofol-induced effects on ETS1 in (A) HGC-27 and (B) AGS cells. miR-195-5p-knockdown reversed the ETS1 protein level in propofol-treated (C) HGC-27 and (D) AGS cells. ${ }^{* * *} \mathrm{P}<0.001,{ }^{* * * *} \mathrm{P}<0.0001$. Circ-PVT1, circular RNA-PVT1; ETS1, E26 oncogene homolog; miR-195-5p, microRNA-195-5p; NC, negative control; anti-miR-195-5p, miR-195-5p inhibitor. 
A

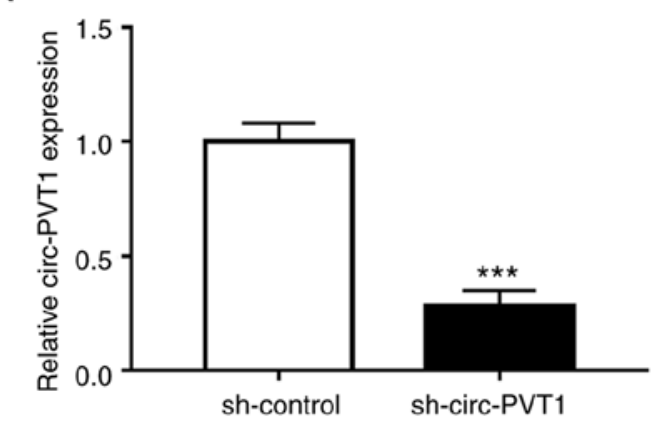

C

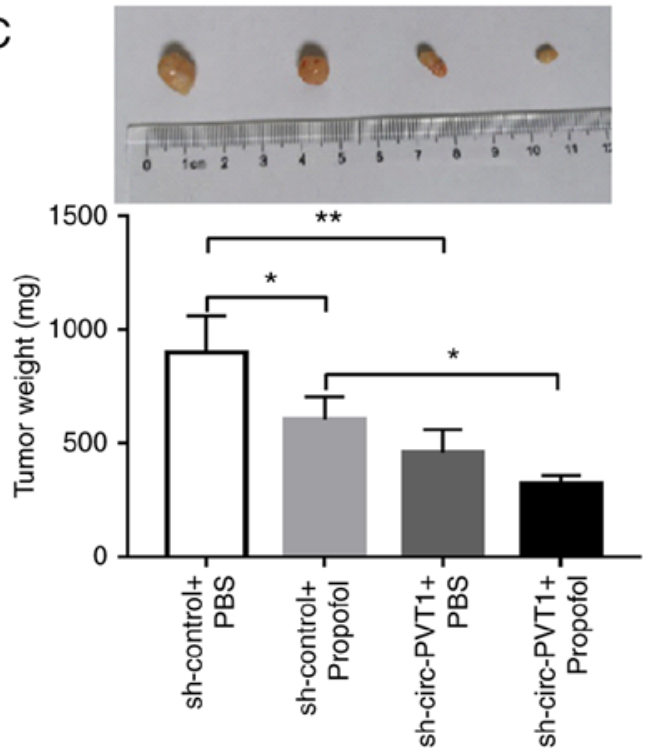

$\mathrm{B}$

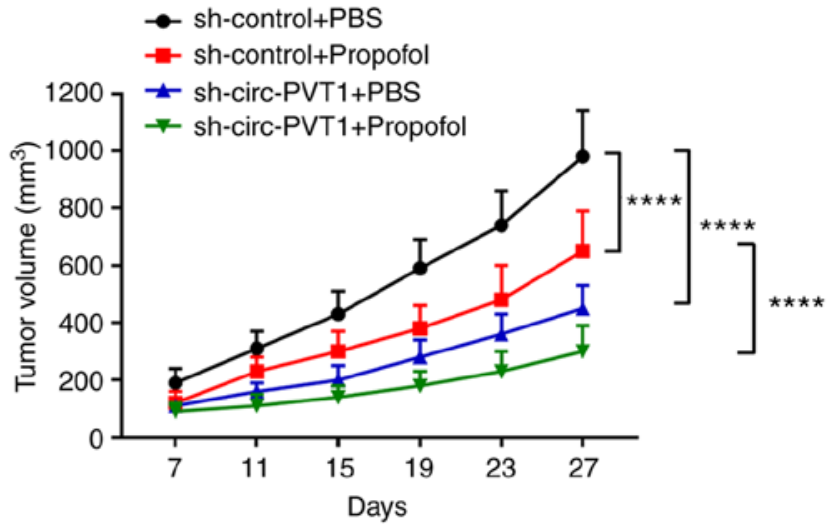

D

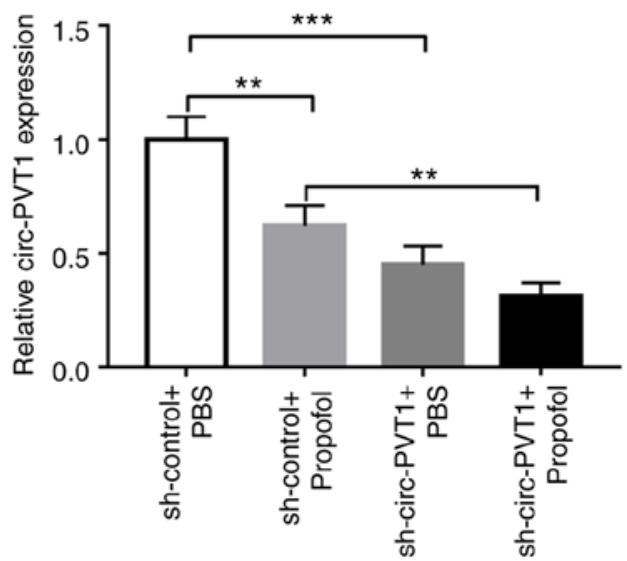

Figure 7. Circ-PVT1-knockdown improves the anti-gastric cancer effect of propofol in vivo. (A) Circ-PVT1 level was detected in AGS cells transfected with sh-control or sh-circ-PVT1 by RT-qPCR. (B) Tumor volume and (C) tumor weight in the sh-circ-PVT1 and sh-control treated groups. (D) Circ-PVT1 expression in tissues was measured by RT-qPCR. ${ }^{*} \mathrm{P}<0.05,{ }^{* *} \mathrm{P}<0.01,{ }^{* * *} \mathrm{P}<0.001,{ }^{* * * *} \mathrm{P}<0.0001$. Circ-PVT1, circular RNA-PVT1; RT-qPCR, reverse transcription-quantitative PCR; sh-circ-PVT1, circular RNA-PVT1 short hairpin RNA.

miR-195-5p in $30 \mathrm{GC}$ tumor tissues (Fig. 5H). The efficiency of transfection with miR-195-5p and anti-miR-195-5p vectors was assessed by RT-qPCR (Fig. 5I). The protein level of ETS1 in HGC-27 and AGS cells was measured by a western blot assay, and the results revealed that the ETS1 level was significantly downregulated by miR-195-5p and significantly upregulated by anti-miR-195-5p (Fig. 5J and K). It was also demonstrated that si-circ-PVT1 and anti-miR-195-5p co-transfection significantly reversed the reduced ETS1 expression induced by si-circ-PVT1 transfection (Fig. 5L and M).

Circ-PVT1 overexpression and miR-195-5p downregulation reverses the propofol-induced effects on ETS1 expression in $G C$ cells. To determine whether propofol exerts its function via the circ-PVT1/miR-195-5p/ETS1 axis, the experimental groups (Control, Propofol, Propofol + pcDNA, and Propofol + circ-PVT1) were designed. As presented in Fig. 6A and B, downregulation of ETS1 induced by propofol in HGC-27 and AGS cells could be restored by circ-PVT1 overexpression. Furthermore, western blotting results suggested that silencing miR-195-5p could abolish the inhibitory action of propofol on ETS1 protein level in HGC-27 (Fig. 6C) and AGS (Fig. 6D) cells.
Circ-PVT1-knockdown improves the anti-GC effect of propofol in vivo. To examine the effectiveness of circ-PVT1-overexpression on tumor growth in vivo, sh-circ-PVT1 stably transfected AGS cells were constructed. The transfection efficiency of sh-circ-PVT1 was examined and presented in Fig. 7A. Subsequently, the transfected AGS cells were inoculated into nude mice, and tumor volume was monitored. The sh-circ-PVT1+ propofol group generated smaller tumors compared with the sh-control + propofol group (Fig. 7B). In addition, the sh-circ-PVT1+ propofol group exhibited the smallest tumor weight among all groups (Fig. 7C). The RT-qPCR results demonstrated that the level of circ-PVT1 in tumors from the sh-circ-PVT1+ propofol group was significantly smaller compared with the sh-control + propofol group (Fig. 7D). These results indicated that circ-PVT1 silencing improves the anti-GC effect of propofol in vivo.

\section{Discussion}

Propofol inhibits cell viability and proliferation, but induces apoptosis of MKN45 GC (40). The present study investigated the GC-suppressive effects of propofol on viability, apoptosis and invasion. It was demonstrated that propofol inhibited 
the proliferation and invasion, but promoted the apoptosis of HGC-27 and AGS cells. It has been reported that P21, Bcl-2 and MMP9 participate in physiological processes. P21 is associated with linking DNA damage to arrest the cell cycle, and $\mathrm{Bcl}-2$ is a member of the Bcl-2 family of regulator proteins that regulate cell death, by either inhibiting or inducing apoptosis $(41,42)$. MMP9 is regarded as a key invasion-associated protein (43). In the present study, propofol could enhance the expression of $\mathrm{P} 21$ and reduce the levels of Bcl-2 and MMP9.

Circ-PVT1 is a cancer susceptibility locus, located on chromosome 8q24 (16). A previous study has demonstrated that circ-PVT1 exhibits a high expression in oral squamous cell carcinoma (OSCC) tissues and cells, which could indicate a potential prognostic importance of circ-PVT1 for patients with OSCC (13). Consistent with the previous study, the current study observed increased circ-PVT1 expression in GC tissues and cells, and the circ-PVT1 level could be decreased by propofol administration in HGC-27 and AGS cells. In addition, in HGC-27 and AGS cells, propofol could restrain the proliferative and invasive abilities, but promoted the apoptotic rate. Furthermore, propofol led to a significant increase in P21 protein level, and a significant decrease in the protein levels of Bcl-2 and MMP9. In a rescue experiment, overexpression of circ-PVT1 reversed the aforementioned effects of propofol.

A study by Yang et al (44) indicated that the expression of miR-195-5p was decreased in breast cancer cells and tissues. The present study predicted and demonstrated that miR-195-5p was a targeted miRNA for circ-PVT1, and miR-195-5p exhibited a low expression in GC tissues and cells. Furthermore, a downregulated level of miR-195-5p restored the tumor suppressor phenotype following si-circ-PVT1 transfection. It was also identified that propofol led to upregulation of miR-195-5p expression. In the rescue experiment, miR-195-5p was knocked down, which revealed that downregulated miR-195-5p reversed the inhibitory effects of propofol on GC.

EST1 is considered to be a pro-oncogene that regulates the pathophysiological progress of GC (45). It has been reported that EST1 is involved in the regulation of proliferation, apoptosis and metastasis, with a higher expression in GC tissues and cells $(46,47)$. In the current study, EST1 was shown to be a downstream target of miR-195-5p. Furthermore, downregulated miR-195-5p restored EST1 expression induced by the downregulation of circ-PVT1. Additionally, downregulation of EST1 by propofol was restored by circ-PVT1 overexpression or miR-195-5p-knockdown.

In the final xenograft experiment, the volume and weight of the tumor decreased significantly following treatment with propofol in the circ-PVT1-knockdown group, and demonstrated a low level of circ-PVT1 expression, which also indicated that the knockdown of circ-PVT1 increased the sensitivity of propofol to tumor suppression.

In conclusion, the present study reported that propofol could inhibit GC progression in vitro and in vivo. Mechanism analysis revealed that propofol suppressed the expression of ETS1 in GC cells by regulating the circ-PVT1/miR-195-5p axis, suggesting that propofol inhibited the GC process through modulating the circ-PVT1/miR-195-5p/ETS1 axis. A limitation of the present study is a small tissue sample size. In addition, ETS1 has been reported to be involved in the PI3K/AKT/mTOR pathway in colorectal cancer cells (48).
Therefore, in future studies, we will focus on whether the regulatory role of circ-PVT1/miR-195-5p/ETS1 on the differentiation and metastasis is mediated by the PI3K/AKT/mTOR pathway in propofol-treated GC cells in vivo.

\section{Acknowledgements}

Not applicable.

\section{Funding}

No funding was received.

\section{Availability of data and materials}

The datasets used and/or analyzed data during the present study are available from the corresponding author on reasonable request.

\section{Authors' contributions}

CZ and ZL designed the study and experiments. ZL and JY generated and analyzed the data. HS and JY performed the experiments and validated the results. HS, CZ and ZL wrote, reviewed and edited the manuscript. All authors read and approved the final manuscript.

\section{Ethics approval and consent to participate}

The present study received approval from the Ethics Committee of The First Affiliated Hospital of Zhengzhou University (Zhengzhou, China). Written informed consent was obtained from each patient.

\section{Patient consent for publication}

Not applicable.

\section{Competing interests}

The authors declare that they have no competing interests.

\section{References}

1. Chidambaran V, Costandi A and D'Mello A: Correction to: Propofol: A review of its role in pediatric anesthesia and sedation. CNS Drugs 32: 873, 2018.

2. Siddiqui RA, Zerouga M, Wu M, Castillo A, Harvey K, Zaloga GP and Stillwell W: Anticancer properties of propofol-docosahexaenoate and propofol-eicosapentaenoate on breast cancer cells. Breast Cancer Res 7: R645-R654, 2005.

3. Cui WY, Liu Y, Zhu YQ, Song T and Wang QS: Propofol induces endoplasmic reticulum (ER) stress and apoptosis in lung cancer cell H460. Tumour Biol 35: 5213-5217, 2014.

4. Wang ZT, Gong HY, Zheng F, Liu DJ and Dong TL: Propofol suppresses proliferation and invasion of pancreatic cancer cells by upregulating microRNA-133a expression. Genet Mol Res 14: 7529-7537, 2015.

5. Wang P, Chen J, Mu LH, Du QH, Niu XH and Zhang MY: Propofol inhibits invasion and enhances paclitaxel-induced apoptosis in ovarian cancer cells through the suppression of the transcription factor slug. Eur Rev Med Pharmacol Sci 17: 1722-1729, 2013.

6. Zhang J, Shan WF, Jin TT, Wu GQ, Xiong XX, Jin HY and Zhu SM: Propofol exerts anti-hepatocellular carcinoma by microvesicle-mediated transfer of miR-142-3p from macrophage to cancer cells. J Transl Med 12: 279, 2014. 
7. Yang C, Gao J, Yan N, Wu B, Ren Y, Li H and Liang J: Propofol inhibits the growth and survival of gastric cancer cells in vitro through the upregulation of ING3. Oncol Rep 37: 587-593, 2017.

8. Peng $Z$ and Zhang Y: Propofol inhibits proliferation and accelerates apoptosis of human gastric cancer cells by regulation of microRNA-451 and MMP-2 expression. Genet Mol Res 15, 2016.

9. Ebbesen KK, Hansen TB and Kjems J: Insights into circular RNA biology. RNA Biol 14: 1035-1045, 2017.

10. Zhang Y, Yang L and Chen LL: Life without A tail: New formats of long noncoding RNAs. Int J Biochem Cell Biol 54: 338-349, 2014.

11. Bonizzato A, Gaffo E, Te Kronnie G and Bortoluzzi S: CircRNAs in hematopoiesis and hematological malignancies. Blood Cancer J 6: e483, 2016.

12. Dykes IM and Emanueli C: Transcriptional and post-transcriptional gene regulation by long non-coding RNA. Genomics Proteomics Bioinformatics 15: 177-186, 2017.

13. He T, Li X, Xie D and Tian L: Overexpressed circPVT1 in oral squamous cell carcinoma promotes proliferation by serving as a miRNA sponge. Mol Med Rep 20: 3509-3518, 2019.

14. Verduci L, Ferraiuolo M, Sacconi A, Ganci F, Vitale J, Colombo T, Paci P, Strano S, Macino G, Rajewsky N and Blandino G: The oncogenic role of circPVT1 in head and neck squamous cell carcinoma is mediated through the mutant p53/YAP/TEAD transcription-competent complex. Genome Biol 18: 237, 2017.

15. Kun-Peng Z, Xiao-Long M and Chun-Lin Z: Overexpressed circPVT1, a potential new circular RNA biomarker, contributes to doxorubicin and cisplatin resistance of osteosarcoma cells by regulating ABCB1. Int J Biol Sci 14: 321-330, 2018.

16. Chen J, Li Y, Zheng Q, Bao C, He J, Chen B, Lyu D, Zheng B $\mathrm{Xu} \mathrm{Y}$, Long Z, et al: Circular RNA profile identifies circPVT1 as a proliferative factor and prognostic marker in gastric cancer. Cancer Lett 388: 208-219, 2017.

17. Liu YY, Zhang LY and Du WZ: Circular RNA circ-PVT1 contributes to paclitaxel resistance of gastric cancer cells through the regulation of ZEB1 expression by sponging miR-124-3p. Biosci Rep 39: BSR20193045, 2019.

18. Pang JC, Kwok WK, Chen Z and Ng HK: Oncogenic role of microRNAs in brain tumors. Acta Neuropathol 117: 599-611, 2009.

19. Mott JL: MicroR NAs involved in tumor suppressor and oncogene pathways: Implications for hepatobiliary neoplasia. Hepatology 50: 630-637, 2009.

20. Tutar Y, Ozgür A, Tutar E, Tutar L, Pulliero A and Izzotti A Regulation of oncogenic genes by MicroRNAs and pseudogenes in human lung cancer. Biomed Pharmacother 83: 1182-1190, 2016.

21. Nie $\mathrm{H}, \mathrm{Mu} \mathrm{J}$, Wang $\mathrm{J}$ and Li Y: miR1955p regulates multidrug resistance of gastric cancer cells via targeting ZNF139. Oncol Rep 40: 1370-1378, 2018

22. Wang F, Ruan L, Yang J, Zhao Q and Wei W: TRIM14 promotes the migration and invasion of gastric cancer by regulating epithelial-to-mesenchymal transition via activation of AKT signaling regulated by miR1955p. Oncol Rep 40: 3273-3284, 2018.

23. Wang J, Li L, Jiang M and Li Y: MicroRNA-195 inhibits human gastric cancer by directly targeting basic fibroblast grow th factor. Clin Transl Oncol 19: 1320-1328, 2017

24. Zhao DL and Wu QL: Effect of inhibition to Yes-related proteins-mediated Wnt $\beta$-catenin signaling pathway through miR-195-5p on apoptosis of gastric cancer cells. Eur Rev Med Pharmacol Sci 23: 6486-6496, 2019

25. Yordy JS and Muise-Helmericks RC: Signal transduction and the Ets family of transcription factors. Oncogene 19: 6503-6513, 2000.

26. Xu D, Wilson TJ, Chan D, De Luca E, Zhou J, Hertzog PJ and Kola I: Ets1 is required for p53 transcriptional activity in UV-induced apoptosis in embryonic stem cells. EMBO J 21 : 4081-4093, 2002.

27. Wolvetang EJ, Wilson TJ, Sanij E, Busciglio J, Hatzistavrou T, Seth A, Hertzog PJ and Kola I: ETS2 overexpression in transgenic models and in Down syndrome predisposes to apoptosis via the p53 pathway. Hum Mol Genet 12: 247-255, 2003.

28. Li X, Lu JY, Zhao LQ, Wang XQ, Liu GL, Liu Z, Zhou CN, Wu M and Liu ZH: Overexpression of ETS2 in human esophageal squamous cell carcinoma. World J Gastroenterol 9: 205-208, 2003.
29. Zhou J, Ng AY, Tymms MJ, Jermiin LS, Seth AK, Thomas RS and Kola I: A novel transcription factor, ELF5, belongs to the ELF subfamily of ETS genes and maps to human chromosome 11p13-15, a region subject to $\mathrm{LOH}$ and rearrangement in human carcinoma cell lines. Oncogene 17: 2719-2732, 1998.

30. Li D, Chen Y, Mei H, Jiao W, Song H, Ye L, Fang E, Wang X, Yang F, Huang K, et al: Ets-1 promoter-associated noncoding RNA regulates the NONO/ERG/Ets-1 axis to drive gastric cancer progression. Oncogene 37: 4871-4886, 2018.

31. Baillat D, Bègue A, Stéhelin D and Aumercier M: ETS-1 transcription factor binds cooperatively to the palindromic head to head ETS-binding sites of the stromelysin-1 promoter by counteracting autoinhibition. J Biol Chem 277: 29386-29398, 2002.

32. Rutter JL, Mitchell TI, Butticè G, Meyers J, Gusella JF, Ozelius LJ, Brinckerhoff CE: A single nucleotide polymorphism in the matrix metalloproteinase-1 promoter creates an Ets binding site and augments transcription. Cancer Res 58: 5321-5325, 1998.

33. Nakada M, Yamashita J, Okada Y and Sato H: Ets-1 positively regulates expression of urokinase-type plasminogen activator (uPA) and invasiveness of astrocytic tumors. J Neuropathol Exp Neurol 58: 329-334, 1999

34. Trojanowska M: Ets factors and regulation of the extracellular matrix. Oncogene 19: 6464-6471, 2000.

35. Liu Z and Klominek J: Regulation of matrix metalloprotease activity in malignant mesothelioma cell lines by growth factors. Thorax 58: 198-203, 2003.

36. Fink K and Boratyński J: The role of metalloproteinases in modification of extracellular matrix in invasive tumor growth, metastasis and angiogenesis. Postepy Hig Med Dosw (Online) 66: 609-628, 2012 (In Polish).

37. Nazir SU, Kumar R, Singh A, Khan A, Tanwar P, Tripathi R, Mehrotra R and Hussain S: Breast cancer invasion and progression by MMP-9 through Ets-1 transcription factor. Gene 711: 143952, 2019.

38. Liu Q, Liu H, Cheng H, Li Y, Li X and Zhu C: Downregulation of long noncoding RNA TUG1 inhibits proliferation and induces apoptosis through the TUG1/miR-142/ZEB2 axis in bladder cancer cells. Onco Targets Ther 10: 2461-2471, 2017.

39. Livak KJ and Schmittgen TD: Analysis of relative gene expression data using real-time quantitative PCR and the 2(-Delta Delta C(T)) method. Methods 25: 402-408, 2001

40. Zhang W, Wang Y, Zhu Z, Zheng Y and Song B: Propofol inhibits proliferation, migration and invasion of gastric cancer cells by up-regulating microRNA-195. Int J Biol Macromol 120: 975-984, 2018.

41. Pisonero-Vaquero S, Soldati C, Cesana M, Ballabio A and Medina DL: TFEB modulates p21/WAF1/CIP1 during the DNA damage response. Cell 9: 1186, 2020.

42. Skommer J, Brittain T and Raychaudhuri S: Bcl-2 inhibits apoptosis by increasing the time-to-death and intrinsic cell-to-cell variations in the mitochondrial pathway of cell death. Apoptosis 15: 1223-1233, 2010.

43. Wang J, Wang C, Li Q, Guo C, Sun W, Zhao D, Jiang S, Hao L, Tian Y, Liu S and Sun MZ: miR-429-CRKL axis regulates clear cell renal cell carcinoma malignant progression through SOS1/MEK/ERK/MMP2/MMP9 pathway. Biomed Pharmacother 127: 110215, 2020.

44. Yang R, Xing L, Zheng X, Sun Y, Wang X and Chen J: The circRNA circAGFG1 acts as a sponge of miR-195-5p to promote triple-negative breast cancer progression through regulating CCNE1 expression. Mol Cancer 18: 4, 2019.

45. Chou NH, Lo YH, Wang KC, Kang CH, Tsai CY and Tsai KW: MiR-193a-5p and $-3 p$ play a distinct role in gastric cancer: miR-193a-3p suppresses gastric cancer cell growth by targeting ETS1 and CCND1. Anticancer Res 38: 3309-3318, 2018

46. Yu Y, Zhang YC, Zhang WZ, Shen LS, Hertzog P, Wilson TJ and Xu DK: Ets1 as a marker of malignant potential in gastric carcinoma. World J Gastroenterol 9: 2154-2159, 2003.

47. Zheng L, Qi T, Yang D, Qi M, Li D, Xiang X, Huang K and Tong Q: microRNA-9 suppresses the proliferation, invasion and metastasis of gastric cancer cells through targeting cyclin D1 and Ets1. PLoS One 8: e55719, 2013.

48. Meng S, Jian Z, Yan X, Li J and Zhang R: LncRNA SNHG6 inhibits cell proliferation and metastasis by targeting ETS1 via the PI3K/AKT/mTOR pathway in colorectal cancer. Mol Med Rep 20: 2541-2548, 2019. 